NEUROGASTROENTEROLOGY

\title{
Influence of body posture on intestinal transit of gas
}

\author{
R Dainese, J Serra, F Azpiroz, J-R Malagelada
}

Gut 2003;52:971-974

See end of article for authors' affiliations

Correspondence to:

Dr F Azpiroz, Digestive

System Research Unit

Hospital General Vall

d'Hebron,

08035-Barcelona, Spain;

fernando.azpiroz@wol.es

Accepted for publication

7 February 2002
Background: Patients describe that body posture may affect their abdominal bloating, distension, and flatulence, but whether changes in position have objectively demonstrable effects, either beneficial or deleterious, has not been investigated.

Aim: To determine the effect of body posture, upright versus supine, on intestinal transit of gas loads. Subjects: Eight healthy subjects without gastrointestinal symptoms.

Methods: In each subject a gas mixture was continuously infused into the jejunum (12 $\mathrm{ml} / \mathrm{min}$ ) for three hours, and gas evacuation, clearance of a non- absorbable gaseous marker, perception, and abdominal girth were measured. Paired studies were randomly performed in each subject on separate days in the upright and supine positions.

Results: In the upright position, intestinal gas retention was much smaller than when supine (13 (52) $\mathrm{ml} v 146(75) \mathrm{ml}$ retention at 60 minutes, respectively; $\mathrm{p}<0.05)$, and clearance of the gas marker was expedited $(72(10) \%$ clearance $v 49(16) \%$ at 60 minutes, respectively; $p<0.05)$. The gas challenge test was well tolerated both in the upright and supine positions without abdominal distension.

Conclusion: Body posture has a significant influence on intestinal gas propulsion: transit is faster in the upright position than when supine.
$\mathrm{P}$ tients with irritable bowel syndrome and functional bloating frequently report that abdominal symptoms, such as bloating and distension that they attribute to intestinal gas, develop progressively during the day and tend to resolve with bed rest. ${ }^{1}$ However, the effect of body posture on intestinal gas transit and evacuation has not been investigated.

As gas within the abdominal cavity tends to float and rise to the top, theoretically caudal gas progression would be facilitated in the supine position. Hence we hypothesised that intestinal gas transit and evacuation are enhanced in the supine compared with the upright position. Using a new technique developed in our laboratory, we compared the effect of body posture, upright versus supine, on gas transit.

\section{MATERIAL AND METHODS}

\section{Participants}

Eight healthy individuals (three women and five men; age range 21-28 years) participated in the study after giving written informed consent. Subjects completed a pre-entry questionnaire to determine the absence of gastrointestinal symptoms, particularly symptoms of constipation, ${ }^{1}$ difficult gas evacuation, feeling of excessive abdominal gas, or excessive gas evacuation. The protocol for the study had been previously approved by the Institutional Review Board of the University Hospital Vall d'Hebron.

\section{Gas transit test}

Jejunal gas infusion

We used a polyvinyl tube assembly (4.1 mm OD) that incorporated a gas infusion channel $(2.0 \mathrm{~mm}$ ID) with multiple side holes scattered over the distal $2 \mathrm{~cm}$ segment. Gas was continuously infused into the proximal jejunum at $12 \mathrm{ml} / \mathrm{min}$, using a modified volumetric pump (Asid Bonz PP 50-300; Lubratronics, Unterschleissheim, Germany). We infused a gas mixture containing $88 \%$ nitrogen, $6.5 \%$ carbon dioxide, and $5.5 \%$ oxygen, bubbled into water for saturation, that mimicked the partial pressures of venous blood gases to minimise diffusion across the intestinal-blood barrier. ${ }^{34} \mathrm{~A}$ non-absorbable stable gaseous marker, $6 \mathrm{ml}$ sulphurhexafluoride $\left(\mathrm{SF}_{6}\right)$, was added to the gas mixture infused during the first 10 minutes. $^{5}$

\section{Rectal gas collection}

To prevent potential effects of the anal sphincters on gas evacuation, gas was collected via an intrarectal catheter (Foley 20 F; Bard, Barcelona, Spain) with the balloon inflated with $5 \mathrm{ml}$ of water. The rectal catheter was connected via a leak proof low resistance collection line to a barostat, ${ }^{67}$ and the volume of gas evacuated was continuously recorded on a paper polygraph (model 6006; Letica, Barcelona, Spain), as previously described. ${ }^{2}$ A sample of gas evacuated (flatus) during each 30 minute period was stored in metallised bags (Gas collection $750 \mathrm{ml}$; QuinTron, Milwaukee, Wisconsin, USA) for later analysis of $\mathrm{SF}_{6}$ concentration by infrared absorbance after determination of standard curves. ${ }^{8}$

\section{Measurement of abdominal girth changes}

A non-stretch $39 \mathrm{~mm}$ wide belt with a metric tape measure was positioned around the abdomen over the umbilicus and adjusted by means of two elastic bands so that girth could be continuously measured. Girth measurements were taken at 15 minute intervals during the study, as follows. Participants were asked to breath in a relaxed manner, maximal and minimal girth changes were determined over three consecutive respiratory excursions (inspiratory and expiratory values), and the average girth value was then calculated.

\section{Perception measurement}

Conscious perception was measured at 15 minute intervals during the studies using a graded questionnaire to score the intensity and type of sensations perceived, and an anatomical questionnaire to measure the location and extension of the sensations. ${ }^{2-12}$ The graded questionnaire included four graphic rating scales graded from 0 (no perception) to 6 (pain) specifically for scoring: (a) pressure/bloating; (b) cramp/ colicky sensation, (c) stinging sensation, and (d) other type of

Abbreviations: $\mathrm{SF}_{6}$, sulphurhexafluoride. 
sensation (to be specified), respectively. Participants were asked to score any abdominal sensation (one or more perceived simultaneously) on the respective scale(s), but only the highest score, instead of the mean or cumulative score, was computed for comparisons. The questionnaire included a tick box (yes/no) to signal belching. The anatomical questionnaire incorporated a diagram of the abdomen divided into nine regions corresponding to the epigastrium, periumbilical area, hypogastrium, both hypochondria, flanks, and iliac fossae. Participants were instructed to mark the locationthat is, abdominal region(s) or extra-abdominal-where the sensations were perceived.

\section{Procedure}

Participants were instructed to follow a diet excluding legumes, vegetables, onion, nuts, cereals, wholemeal bread, and fizzy drinks during the two days prior to each study day. The night before the study they had a dinner that could consist of meat, fish, eggs, rice, pasta, and/or white bread but avoiding in particular dairy products, salad, fruit, and alcoholic beverages. All participants were required to have one bowel movement within the 12 hours prior to the study or otherwise the study was postponed. On each study day participants were orally intubated after an eight hour fast. The intestinal tube assembly was positioned under fluoroscopic control with the gas infusion ports $5 \mathrm{~cm}$ caudad to the angle of Treitz, the gas collection catheter was introduced into the rectum. The studies were conducted in a quiet isolated room. In each experiment, intestinal gas was infused continuously for three hours, and perception and girth measurements were obtained at 15 minute intervals.

\section{Experimental design}

In each subject two experiments were performed in random order on different days separated by an interval of at least one week: (a) while placed supine in bed at an angle of $30^{\circ}$ to the horizontal and (b) while standing with the arms on a $1.12 \mathrm{~m}$ high table that provided firm support to rest on it. In the standing experiments, subjects were allowed to sit down for five minutes at 20 minute intervals.

\section{Outcome measures}

In each subject, we calculated the volume of gas retained within the gut as the difference between the volume of gas infused and the volume of gas recovered. Perception of abdominal sensations experienced by participants during the studies was measured by the score rated in the scales. In each subject, we also counted the number of times each abdominal sensation was scored to calculate the frequency (as per cent distribution) of each specific sensation. In the anatomical questionnaire, we calculated the percentage of sensations referred over each abdominal region as well as the percentage referred over more than one region.

\section{Statistical analysis}

For each subject, repeat measurements of gas retention, abdominal perception, and girth changes were averaged over 30 minute periods. In each experimental condition (upright position and supine) mean values (SEM) of the parameters measured in the group of subjects were calculated. The Komolgorov-Smirnov test was used to check the normality of data distribution. Paired comparisons were performed by the Student's $t$ test for parametric normally distributed data, or otherwise by the Wilcoxon signed rank test. Correlations between paired data were examined by linear regression analysis.

\section{RESULTS}

\section{Gas transit and clearance}

In the supine position, the gas infused was initially retained within the gut. There was a lag time before subjects started to

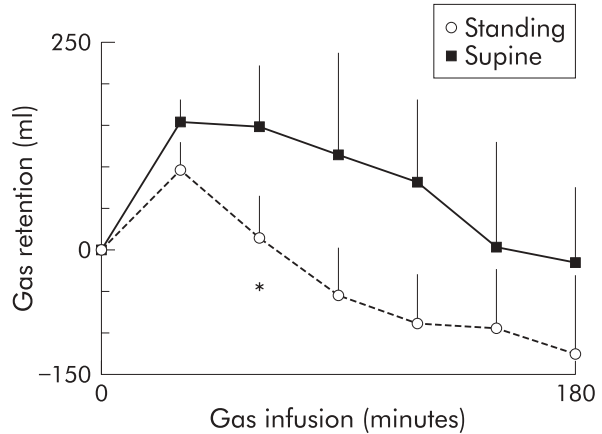

Figure 1 Effect of body position on gas transit. Gas retention measured as gas infused minus evacuated over 180 minutes of jejunal gas infusion. During the first 60 minute period, infused gas was retained in the supine but not in the upright position ( $\left.{ }^{*} p<0.05\right)$.

evacuate gas (the first $10 \mathrm{ml}$ of gas were collected from the rectum 17 (5) minutes after starting the infusion) and therefore a significant volume of gas was initially retained within the gut. Specifically, during the first 30 minutes, 47 (14)\% of the volume infused was retained. After one hour, gas evacuation increased to match and even exceed the infusion rate and consequently the volume of gas retained progressively decreased (fig 1). By the end of the three hour experiment, the total volume of gas infused had been evacuated. Gas retention was statistically significant only during the first 60 minutes of gas infusion.

In the upright position, gas transit and evacuation were faster than in the supine position, and the first $10 \mathrm{ml}$ of gas were collected from the rectum 10 (2) minutes after starting the infusion $(\mathrm{p}<0.05 v$ supine $)$. During the first 30 minutes, only $23(10) \%$ of the volume infused was retained in the gut $(\mathrm{p}<0.05 v$ supine) but this initial retention was rapidly cleared out from the gut. By the end of the study period, gas retention was negative-that is, the total volume of gas evacuated (endogenous plus exogenous) was greater than the volume of gas infused. Gas retention was overall smaller in the upright than in the supine position but the differences were only statistically significant during the first hour when the volume of gas retained in the supine position was significant (mean retention $53(41) \mathrm{ml}$ in the upright $v 149(51) \mathrm{ml}$ in the supine position; $\mathrm{p}<0.05$ ).

Evidence of faster gas transit in the upright position was further supported by a significantly faster $\mathrm{SF}_{6}$ clearance (fig 2 ). $\mathrm{SF}_{6}$ started to be evacuated significantly faster in the upright than in the supine position (34 (4) $v 49$ (8) minutes, respectively; $\mathrm{p}<0.05$ ). By one hour, per cent recovery of $\mathrm{SF}_{6}$ was significantly higher in the upright than in the supine position (fig 2). By the end of the studies, $\mathrm{SF}_{6}$ recovery was virtually complete in both experimental conditions (97 (2)\% upright and in 98 (1)\% supine).

\section{Perception and abdominal distension}

All subjects tolerated the gas infusion with minimal perception either in the upright or supine position (fig 3), and despite the different retention volumes, perception scores were similar in both positions (mean perception score $0.7(0.2)$ in the supine and $0.6(0.2)$ in the upright position; NS). Abdominal girth measured in the same subjects on different experimental days before starting the gas infusion was 771 (31) $\mathrm{mm}$ in the upright position and 779 (31) $\mathrm{mm}$ in the supine position. Girth remained constant over time during the gas infusion tests (no significant changes were observed) and was not affected by the relatively small volumes of intraluminal gas in the upright or supine position (mean girth change 2 (1) mm and 3 (1) $\mathrm{mm}$, respectively; NS). The type of sensations acknowledged by participants was similar in both positions: the incidence of pressure/bloating and cramp/colicky sensation was $44(17) \%$ and 54 (17)\% in the upright position versus 


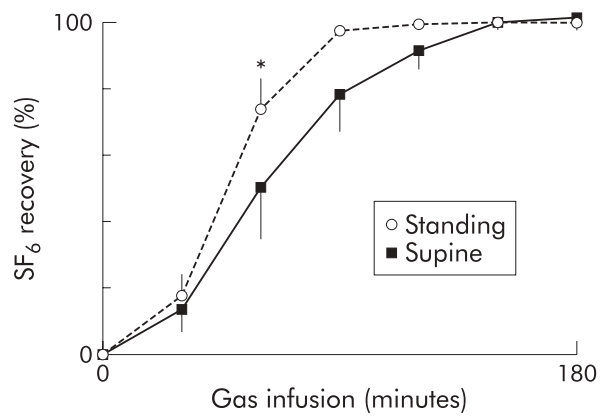

Figure 2 Effect of body position on gas clearance. Clearance of a sulphurhexafluoride $\left(\mathrm{SF}_{6}\right)$ jejunal bolus was significantly faster in the upright than in the supine position ( ${ }^{*} p<0.05$ at 60 minutes) but by 180 minutes marker recovery was complete.

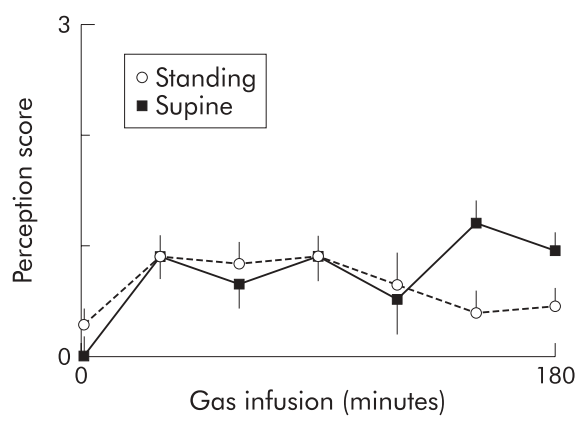

Figure 3 Effect of body position on abdominal perception. Perception during jejunal gas infusion was scored from 0 to 6 (only 0-3 score scale shown). Gas infusion was equally well tolerated both in the upright and supine positions (NS).

$26(14) \%$ and $72(14) \%$ in the supine position, respectively (NS), and these sensations were predominantly referred to the abdominal midline (86 (6)\% and $84(8) \%$, respectively), and circumscribed to a single abdominal area $(52(14) \%$ and 69 (13)\%, respectively).

\section{DISCUSSION}

Our data indicate that body position has a significant influence on intestinal gas transit. Specifically, we have shown that gas transit and evacuation are faster in the upright than in the supine position.

As previously observed, ${ }^{2}$ gas infusion into the jejunum was well tolerated by healthy subjects. In the supine position, infused gas was initially retained in the gut but after one hour the intestine cleared out the retained gas, a pattern previously observed under similar experimental conditions. ${ }^{13}$ Contrary to our initial hypothesis, intestinal gas clearance was even more efficient in the upright position.

Due to the lack of solid data, we elaborated our hypothesis on purely theoretical speculative reasoning. It has been shown that gastric emptying of a saline test meal is faster in the right than in the left lateral decubitus, and an effect of gravity was suggested.$^{14}{ }^{15}$ Furthermore, gastric emptying of the lipid component of a meal, which tends to float within the intragastric contents due to its lower density, is slower than the aqueous phase. ${ }^{16}$ As gas tends to rise to the top within the abdominal cavity, ${ }^{17}$ we extrapolated these data, and postulated that caudad propulsion and evacuation could be impaired in the upright position. Contrary to such speculation that placed undue weight on gravitational forces, the gas behaviour observed in the upright position probably reflects active motor activity of the gut that effectively propulses gas against the passive resistive forces.

Several lines of evidence indicate that the motor activity of the intestinal wall propels intestinal gas in the caudad direc- tion. It has previously been shown that the amount of gas within the gut is normally approximately $200 \mathrm{ml} .{ }^{18}{ }^{19}$ In a dose-response study with our gas challenge test, we showed that healthy subjects propulse and evacuate as much gas as infused without retention, ${ }^{2}$ which suggests either that the intestinal capacity is fixed and any gas excess passively overflows or, more likely, that intestinal propulsion is finely adapted to the luminal gas load to maintain gas homeostasis. Further data substantiate the latter possibility by showing, in the first instance, that pharmacological modulation of gut motor activity modifies gas transit. Specifically, administration of glucagon, which inhibits gut motor activity, markedly delays gas transit, ${ }^{20}$ and conversely, in patients with gas retention, neostigmine administration exerts a marked prokinetic effect that results in gas clearance. ${ }^{21}$ Furthermore, gut stimuli known to induce motor reflexes also modulate gas propulsion. For instance, intraluminal lipids dose dependently delay gas transit ${ }^{22}$ whereas focal gut distension accelerates transit. ${ }^{1323}$ Body posture, and particularly the upright position in comparison with the supine position, involves changes both in abdominoperineal muscle tone and in the intra-abdominal hydrostatic pressure gradient, and results in a marked pressure increment in the lower abdominal cavity. Theoretically, abdominal pressure could not exert direct effects on intraluminal gas movement, except at the rectal venting site. However, these changes may activate mechanoreceptors at various possible sites and conceivably modify intestinal gas propulsion via somatovisceral reflexes. The anus effectively controls gas evacuation ${ }^{20}$ but the use of an intrarectal gas collection catheter convincingly ruled out potential effects of the anal sphincters.

Our data may have substantial pathophysiological implications because they provide an experimentally controlled answer to a relevant clinical question. Our results imply that the upright position would favour gas evacuation in patients with gas retention. As our studies were conducted in healthy volunteers, we cannot affirm or deny such a prediction. However, in patients complaining of abdominal bloating and distension without apparent cause, reflex control of intestinal gas propulsion is impaired. ${ }^{22}{ }^{24}$ Therefore, it is possible that reflex motor activity triggered by the upright position also fails and constitutes an additional contributory factor to gas retention.

\section{ACKNOWLEDGEMENTS}

Supported in part by the Spanish Ministry of Education (Dirección General de Enseñanza Superior del Ministerio de Educación y Cultura, PM97-0096 and BSA 2001-2584) and the National Institutes of Health of the United States (grant DK 57064). Dr Dainese was supported by a scholarship from the University of Padova. The authors thank Anna Aparici and Maite Casaus for technical support, and Gloria Santaliestra for secretarial assistance.

\section{Authors' affiliations}

R Dainese*, J Serra, F Azpiroz, J-R Malagelada, Digestive System Research Unit, University Hospital Vall d'Hebron, Autonomous University of Barcelona, Barcelona, Spain

*Present address: Section of Gastroenterology, Department of Surgical and Gastroenterological Science, University of Padova, 35128 Padova, Italy

\section{REFERENCES}

1 Thompson WG, Longstreth G, Drossman DA, et al. Functional bowel disorders and D. Functional abdominal pain. In: Drossman DA Corazziari E, Talley NJ, et al, eds. The Functional Gastrointestinal Corazziari E, Talley N, et al, eds. The Functional Gastrointestind
Disorders. McLean, VA: Degnon Associates, 2000:351-432.

2 Serra J, Azpiroz F, Malagelada JR. Intestinal gas dynamics and tolerance in humans. Gastroenterology 1998;115:542-50.

3 Foster RE. Physiological basis of gas exchange in the gut. Ann NY Acad Sci 1968;150:4-12.

4 Pogrund RS, Steggerda FR. Influence of gaseous transfer between the Pogrund RS, Steggerda FR. Influence of gaseous transfer between the
colon and blood stream on percentage gas compositions of intestinal flatus in man. Am J Physiol 1948;153:475-82. 
5 Levitt MD. Production and excretion of hydrogen gas in man. N Engl J Med 1969.281:122-7.

6 Azpiroz F, Malagelada JR. Gastric tone measured by an electronic barostat in health and postsurgical gastroparesis. Gastroenterology 1987:92:934-43

7 Azpiroz F, Malagelada JR. Physiological variations in canine gastric tone measured by an electronic barostat. Am J Physiol

1985;248:G229-37.

8 Jonmarker C, Castor R, Drefeldt B, et al. An analyzer for in-line measurement of expiratory sulfur hexafluoride concentration. Anesthesiology 1985;63:84-8.

9 Serra J, Azpiroz F, Malagelada JR. Perception and reflex responses to intestinal distention in humans are modified by simultaneous or previous stimulation. Gastroenterology 1995;109:1742-9.

10 Serra J, Azpiroz F, Malagelada JR. Modulation of gut perception in humans by spatial summation phenomena. J Physiol 1998;506:579-87.

11 Azpiroz F. Gastrointestinal perception: pathophysiological implications. Neurogastroenterol Motil 2002;14:229-39

12 Gracely R. Studies of pain in normal man. In: Wall P, Melzac R, eds. Textbook of Pain. Edinbourgh: Churchill Livingstone, 1994:315-36.

13 Serra J, Azpiroz F, Malagelada JR. Gastric distension and duodenal lipid infusion modulate intestinal gas transit and tolerance in humans. Am J Gastroenterol 2002;97:2225-30.

14 Burn-Murdoch R, Fisher MA, Hunt JN. Does lying on the right side increase the rate of gastric emptying? J Physiol 1980;302:395-8.
15 Anvari $M$, Horowitz M, Fraser $R$, et al. Effects of posture on gastric emptying on nonnutrient liquids and antropyloroduodenal motility. Am J Physiol 1995:268:G868-71.

16 Cortot A, Phillips S, Malagelada JR. Gastric emptying of lipids after ingestion of a solid-liquid meal in humans. Gastroenterology 1981;80:922-7.

17 Hood JH. Effect of posture on the amount and distribution of gas in the intestinal tract of infants and young children. Lancet 1964:II: 107-10.

18 Levitt MD. Volume and composition of human intestinal gas determined by means of an intestinal washout technic. N Engl J Med 1971;284:1394-8

19 Serra J, Azpiroz F, Malagelada JR. Impaired transit and tolerance of intestinal gas in the irritable bowel syndrome. Gut 2001;48:14-19.

20 Serra J, Azpiroz F, Malagelada JR. Mechanisms of intestinal gas retention in humans: impaired propulsion versus obstructed evacuation. Am J Physiol Gastrointest Liver Physiol 2001;281:G138-43.

21 Caldarella $M$, Serra J, Azpiroz F, et al. Prokinetic effects in patients with intestinal gas retention. Gastroenterology 2002;122:1748-55.

22 Serra J, Salvioli B, Azpiroz F, et al. Lipid-induced intestinal gas retention in the irritable bowel syndrome. Gastroenterology 2002;123:700-6.

23 Harder H, Serra J, Azpiroz F, et al. Reflex control of intestinal gas dynamics and tolerance. Gastroenterology 2000;1 18:A689.

24 Passos MC, Serra J, Azpiroz F, et al. Impaired reflex control of intestinal gas propulsion in patients with abdominal bloating. Gastroenterology 2002;122:A549. 